\title{
Simple Formulas for Improved Point- Charge Electrostatics in Classical Force Fields and Hybrid Quantum Mechanical/Molecular Mechanical Embedding
}

\author{
G. A. CISNEROS, ${ }^{1}$ S. NA-IM THOLANDER, ${ }^{2,3}$ O. PARISEL, ${ }^{2,3}$ \\ T. A. DARDEN, ${ }^{1}$ D. ELKING ${ }^{1}{ }^{1}$ L. PERERA ${ }^{1}{ }^{1}$ J.-P. PIQUEMAL ${ }^{2,3}$ \\ ${ }^{1}$ Laboratory of Structural Biology, National Institute of Environmental Health Sciences, \\ P.O. Box 12233, MD F0-08, 111 TW. Alexander Dr., NC 27709 \\ ${ }^{2}$ UPMC Univ Paris 06, UMR 7616, Laboratoire de Chimie Théorique, Case Courrier 137, \\ 4 Place Jussieu, F-75005 Paris, France \\ ${ }^{3}$ CNRS, UMR 7616, Laboratoire de Chimie Théorique, Case Courrier 137, 4 Place Jussieu, \\ F-75005 Paris, France
}

Received 21 February 2008; accepted 27 February 2008

Published online 21 April 2008 in Wiley InterScience (www.interscience.wiley.com). DOI 10.1002/qua.21675

\begin{abstract}
We present a simple damping scheme for point-charge electrostatics that could be used directly in classical force fields. The approach acts at the charge (or monopole) level only and allows the inclusion of short-range electrostatic penetration effects at a very low cost. Results are compared with density functional theory Coulomb intermolecular interaction energies and with several other methods such as distributed multipoles, damped distributed multipoles, and transferable Hermite-Gaussian densities. Realistic trends in the interactions are observed for atom-centered MertzKollman corrected point-charge distributions. The approach allows increasing the selectivity of parameters in the case of metal complexes. In addition, two QM/MM calculations are presented where the damping function is employed to include the MM atoms located at the QM/MM boundary. The first calculation corresponds to the gasphase proton transfer of aspartic acid through water and the second is the first step of the reaction catalyzed by the 4-oxalocrotonate tautomerase (4OT) enzyme. First, improved agreement is observed when using the damping approach compared with the conventional excluded charge method or when including all charges in the calculation.
\end{abstract}

Correspondence to: J.-P. Piquemal; e-mail: jpp@lct.jussieu.fr

Contract grant sponsors: NIH, NIEHS. 
Second, in the case of $4 \mathrm{OT}$, the damped charge approach is in agreement with previous calculations, whereas including all charges gives a significantly higher energy barrier. In both cases, no reparameterization of the van der Waals part of the force field was performed. (c) 2008 Wiley Periodicals, Inc. Int J Quantum Chem 108: 1905-1912, 2008

Key words: electrostatics; force fields; QM/MM; embedding; penetration energy; reaction path

\section{Introduction}

$\mathbf{F}$ or over 30 years, most of force fields such as AMBER [1], CHARMM [2], or GROMOS [3] have chosen a classical point-charge representation of electrostatics. Such representation has been refined over the last decade because of a direct fit of ab initio electrostatic potentials (ESP) [4, 5]. Coupled to efficient periodic boundary conditions techniques $[6,7]$, they have enabled long molecular dynamics simulations and have been convenient to the implementation of hybrid Quantum Mechani$\mathrm{cal} /$ Molecular Mechanical (QM/MM) approach [8, 9]. More elaborate distributed multipole representations of the charge distribution [10-13] are also available and have been successfully used in the framework of new generation polarizable force fields [14-20]. Nevertheless, despite these sophistications, all approaches only approximate the longrange "multipolar" part of the ab initio intermolecular Coulomb interaction energy and neglect the so-called "penetration energy", an overlap term arising in the ab initio reference calculations. In that context, several strategies have been explored in order to include such effects. Some approaches such as Sum of Interaction Between Fragment $\mathrm{Ab}$ initio (SIBFA; the updated version for the SIBFA program can be obtained from the authors) $[14,15]$ or the Effective Fragment Potential (EFP) [19] use damping functions [14, 20-22] coupled to distributed multipoles whereas others employ a simplified charge density treatment [23-25]. Among these latter methods, the Gaussian Electrostatic Model (GEM) [12, 15, 26-28], a new generation force field based on density fitting, uses a quasi-exact representation of electrostatics based on Hermite-Gaussian functions. This force field allows including long-range and short-range effects and has demonstrated the central importance of electrostatic penetration effects in the reproduction of quantum chemistry results within the framework of QM/MM embedding [27].

Relying on that work, we chose in this article to explore "simple" improvements of the electrostatic interaction energy treatments in classical nonpolarizable force fields and QM/MM using ESP-fitted point charges. To do so, we first detail a new damped charge approach and test the methodology on several complexes, including 10 stationary points on the water dimer energy [29] surface, two water clusters, and several water-metal complexes.

Subsequently, we explore the role of damped charges at the QM/MM boundary for two model reactions. An issue in $\mathrm{QM} / \mathrm{MM}$ calculations is the boundary between the subsystems. In general the point charges associated with the MM atoms bonded to the QM/MM boundary atoms are zeroed out in order to avoid overpolarization of the QM wave function. However, it has been shown that this can have an impact on the results [30]. One way to overcome this problem is to "blur" the charges with a Gaussian function [31]. Here we explore the possibility of using damped charges instead, to improve the QM/MM boundary description. Note that other effects such as long-range electrostatics and polarization also play an important role $[32,33]$. These effects are not considered in this work.

The investigated reactions are the gas phase proton transfer of aspartic acid through water and the first step of the reaction catalyzed by 4-oxalocrotonate tautomerase (4OT). In these two cases, we compare the damped charge results to conventional excluded charge results, as well as to results where all charges have been included for the QM/MM calculation (for the aspartic acid reaction).

\section{Computational Details}

\section{REFERENCE CALCULATIONS}

Constrained space orbital variations (CSOV) [34, 35] density functional theory reference electrostatic interaction energies have been computed using an in-house [36a] version of HONDO 95.3 [36b]. We have used the B3LYP $[37,38]$ functional coupled to the aug-cc-pVTZ basis set [39a] for all atoms except for metals $[\mathrm{Mg}(\mathrm{II}), \mathrm{Zn}(\mathrm{II})$, and $\mathrm{Cu}(\mathrm{I})$ cations] which 
the $6-31 G^{* *}[39 \mathrm{~b}]$ basis set was used. Mertz-Kollman point charges [4] have been derived at the same level of theory using Gaussian 03 [40]. Damped and undamped multipole interactions have been performed using the SIBFA package $[15 a, b]$.

\section{DAMPING SCHEMES}

The classical formula for point-charge electrostatics is given below in a.u.:

$$
E=\left(q_{i} q_{j}\right) / r_{i j}
$$

where $r_{i j}$ is the distance between atoms $i$ and $j$ bearing the charges.

It can be simply modified by replacing the charges by their damped value following:

$$
E=\left(q_{i}^{*} q_{j}^{*}\right) / r_{i j}
$$

where

$$
q_{i}^{*}=2 q_{i}-\left(Z_{i}-\left(Z_{i}-q_{i}\right)\left(1-\exp \left(\Omega_{i j}\right)\right)\right)
$$

Here $Z_{i}$ is the number of valence electrons of atom $i, \Omega_{i j}$ is a factor depending on the distance between atoms $i$ and $j$ and $q_{i}$ denotes the charge of atom $i$. Several simple expressions can be derived for $\Omega_{i j}$, we will here explore two of them:

$$
\Omega_{i j}=\frac{\lambda_{\mathrm{AB}} r_{i j}}{\frac{\left(Z_{i}-q_{i}\right)}{Z_{i}}}
$$

where $\lambda_{\mathrm{AB}}$ is an adjustable parameter depending on the nature of the two interacting molecules $A$ and $B$, $Z_{i}$ is the number of valence electron of atom $i$, and $q_{i}$ is its charge.

$$
\Omega_{i j}=\frac{\lambda_{\mathrm{AB}} r_{i j}}{R_{\mathrm{vdw}}(i)+R_{\mathrm{vdw}}(j)}
$$

$\lambda_{\mathrm{AB}}$ is a transferable parameter, adjusted on waterwater interactions and kept constant for all calculations. $\lambda_{\mathrm{AB}}$ is modulated by the $R_{\mathrm{vdw}}$ parameters which are effective van der Waals radius parameters $(\AA)$ specific for a given atom $i$ or $j$. The values of the $\lambda_{\mathrm{AB}}$ and $R_{\mathrm{vdw}}$ parameters are given in the text.

\section{QM/MM DETAILS}

All QM/MM calculations were performed using a modified version of Gaussian 03 [40] interfaced to a modified version of TINKER [41]. The AMBER95 all-atom force field parameter set [42] and the TIP3P model for water [43] were used. In both cases we have used the pseudobond approach to model the $\mathrm{QM} / \mathrm{MM}$ boundary $[44,45]$. All geometry optimizations were performed at the HF/3-21G level following the iterative minimization procedure described by Zhang et al. [46]. All path optimization calculations were performed with the quadratic string method (QSM) as proposed by Burger and Yang [47]. The steepest descent results were obtained with the quadratic synchronous transit (QST3) algorithm of Gonzalez and Schlegel [48]. In all cases, the reactant and product (or intermediate for 4OT) of each path calculation were fully optimized using the default convergence in Gaussian 03 for the QM subsystem and a $0.2 \mathrm{kcal} \mathrm{mol}^{-1} \AA^{-1}$ threshold for the MM gradient convergence. In the case of the optimizations that included the damped charges, the MM atoms with damped charges were frozen during the optimization procedure. After obtaining the end points, the paths were optimized using the iterative QSM scheme [47] until the MM environment converged to the same criteria as above for all points along the path. The QM/MM boundary was modeled using pseudobonds [44, 45] and damped charges were included in calculations as explained later.

\section{Results and Discussion}

\section{INTERACTION ENERGIES: FROM WATER TO METALS}

Both expressions of $\Omega_{i j}$ have been tested and appear to be quite equivalent for a given complex.

Expression 4 is very simple as it embodies a single parameter only. Nevertheless, it requires a parameterization of the sole parameter $\lambda_{\mathrm{AB}}$ which is specific for a given pair of interacting molecules. On the other hand, expression 5 should be transferable as $\lambda_{\mathrm{AB}}$ is found once and for all after fitting on a set of water dimers and can be extended to any system by parameterizing additional effective radii in the same spirit of the multipole damping function. For all calculations presented in this section, $\lambda_{\mathrm{AB}}$ will be fixed to -3.34 , a value adjusted on water dimers. 


\section{Dimers and Clusters of Water Molecules}

Van der Waals radii were chosen to be 1.56 and $1.2 \AA$ for oxygen and hydrogen atoms, respectively, following Ref. [20].

Figure 1 displays a comparison of different approaches including CSOV/B3LYP reference computations for intermolecular electrostatics in 10 stationary points on the water dimer potential-energy surface [29]. The damped approach [Eq. (5)] considerably reduces the error of the point-charge approach compared with references CSOV/B3LYP computations. Indeed, an average error of 0.48 $\mathrm{kcal} / \mathrm{mol}$ is observed on the 10 dimers which reduce the average error by a factor of 4 , i.e., 1.88 $\mathrm{kcal} / \mathrm{mol}$ with respect to naked point charges. In fact, we can rank the damped and undamped pointcharges approaches compared with existing methodologies such as GEM's Hermite densities, VignéMaeder-Claverie distributed multipoles, damped distributed multipoles by computing electrostatics as follows:

$$
\begin{aligned}
\text { DFT }>\text { GEM }>\text { Damped multipoles } & \\
>\text { Damped point charges } & >\text { Multipoles } \\
& >\text { Point charges }
\end{aligned}
$$

This ranking clearly shows that the Hermite densities and damped multipoles are in better agreement with CSOV than the damped charges. However, the damped atom-centered point charges perform surprisingly better than distributed multipoles. This is due to the fact that naked distributed multipoles do not include the penetration energy which appears clearly nonnegligible compared with the ab initio results.

We have also tested our damped point-charges approach on the two water clusters for which ab initio electrostatics were available at the same level [26]. We obtain a satisfactory transferability as damped point-charge electrostatics was found to be about $-192.08 \mathrm{kcal} / \mathrm{mol}$ (compared with -186.84 $\mathrm{kcal} / \mathrm{mol}$ for CSOV) and about $-313.3 \mathrm{kcal} / \mathrm{mol}$ (compared with $-309.38 \mathrm{kcal} / \mathrm{mol}$ for CSOV) for the 16 and 20 water molecule clusters, respectively.

\section{Water-Metal Complexes}

Scans of the metal cation-water oxygen distance have been performed in several $C_{2 v}$ metal(II)-water complexes (see Fig. 2). As seen in Table I, the approach is also performing well for metals as the damping allows including a specific parameterization of the +2 charge. $\mathrm{Ca}(\mathrm{II}), \mathrm{Mg}$ (II), and $\mathrm{Zn}$ (II) electrostatic interactions with water appear clearly different at a given O-Metal distance amounting to $1.9 \AA$, in agreement with ab initio computations. Conversely, simple point charges cannot discriminate the cations, thus giving in all cases an identical electrostatic energy of $-46.53 \mathrm{kcal} / \mathrm{mol}$. It is important to point out that such treatment could allow including relativistic effects in electrostatic by means of scalar relativistic pseudopotentials [36c,d] if aiming at considering heavy metals. As for the multipole damping function, the approach converges to the classical point-charge approximation
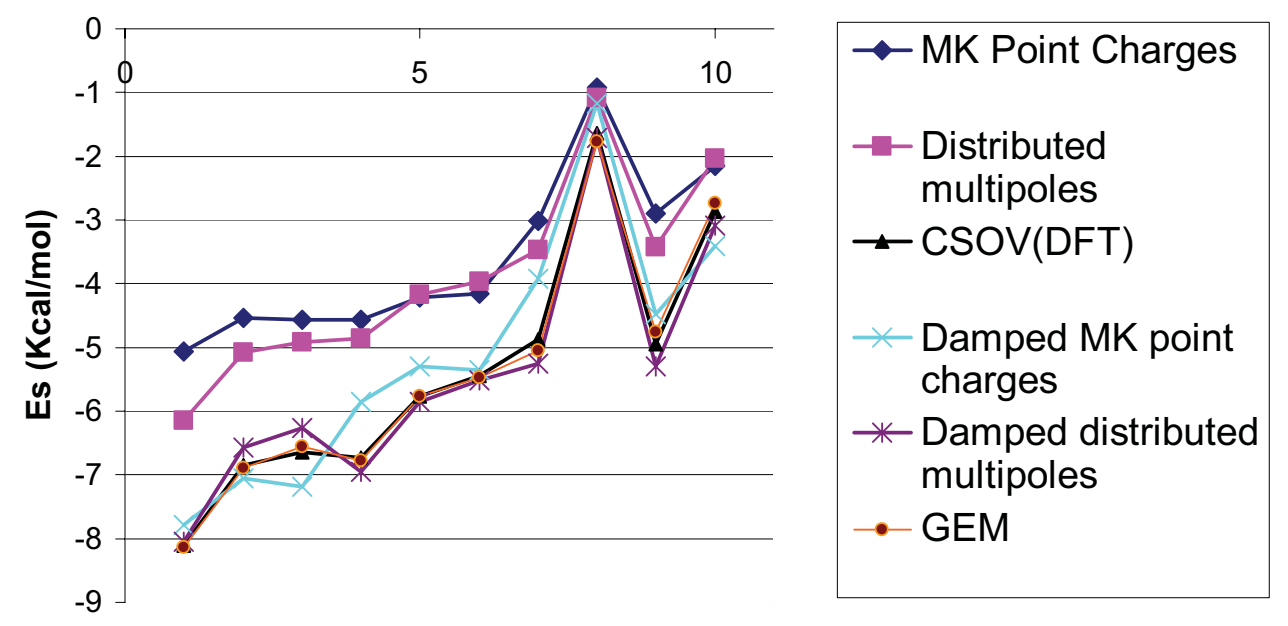

FIGURE 1. Coulomb interaction for the 10 water dimers using different methods. [Color figure can be viewed in the online issue, which is available at www.interscience.wiley.com.] 


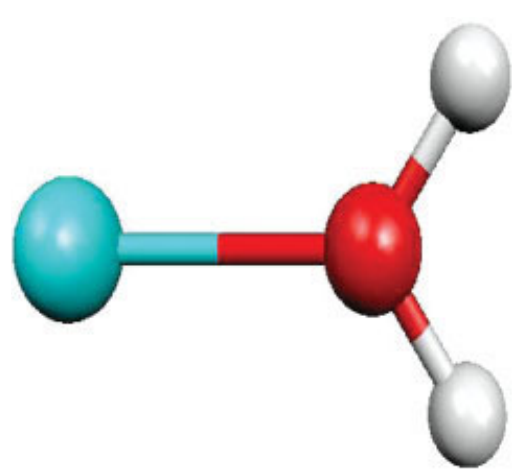

FIGURE 2. Water- $\mathrm{M}^{2+}$ complex orientation ( $\mathrm{M}=\mathrm{Ca}$, $\mathrm{Mg}, \mathrm{Zn}$ ). [Color figure can be viewed in the online issue, which is available at www.interscience.wiley.com.]

at long range, in close agreement with ab initio benchmarks as penetration effects vanish.

\section{QM/MM}

Two reactions were considered in order to test how the charges obtained with the damping function perform in $\mathrm{QM} / \mathrm{MM}$ calculations. The additional effective radii (different from $\mathrm{O}$ and $\mathrm{H}$ ) were taken from Ref. [20]. No reparameterization of the force field's van der Waals parameters was made. The first reaction tested is the gas phase proton transfer of aspartic acid to water. In this case, five calculations were performed: in the first two, all atoms are treated quantum mechanically, while for the remaining three only the side-chain of Asp and the water molecule are included in the QM region. The reactions were treated as follows: (1) a QST3 calculation with all atoms treated at the QM level to determine the correct energy barrier; (2) a QSM path optimization with all atoms treated at the QM level; (3) a QM/MM QSM path optimization with excluded charges [46]; (4) QM/MM QSM path optimization including all charges in the MM sub-

\section{TABLE I}

Comparison of CSOV electrostatics to damped charge results for a water molecule interacting with a metal cation at a distance of $1.9 \AA$ (Fig. 1).

\begin{tabular}{lccc}
\hline Method & $\mathrm{Ca}(\mathrm{II})$ & $\mathrm{Mg}(\mathrm{II})$ & $\mathrm{Zn}(\mathrm{II})$ \\
\hline CSOV & -83.81 & -64.54 & -76.39 \\
Damped charge & -82.7 & -65.71 & -75.36 \\
vdw & 1.4 & 1.62 & 1.5 \\
\hline
\end{tabular}

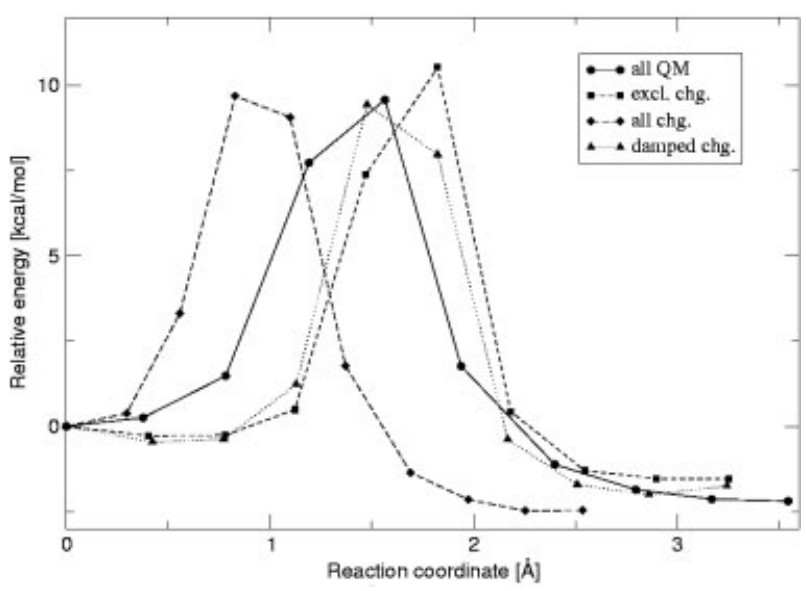

FIGURE 3. Calculated paths for the Asp reaction.

system; and (5) a QM/MM QSM path optimization including all charges with damped charges on the atoms directly bonded to the pseudoatom. In this case, the used $\lambda_{\mathrm{AB}}$ for the damping function [Eq. (5)] was -7.0 . Indeed, $\lambda_{\mathrm{AB}}$ needs to be reparameterized in order to also include damping for the intramolecular charge interactions (only intermolecular interactions were considered previously) and to overcome the lack of explicit force-field polarization treatment at the boundary. An energy barrier of $10 \mathrm{kcal} / \mathrm{mol}$ is obtained with QST3 for the Asp proton transfer, compared with $9.6 \mathrm{kcal} / \mathrm{mol} \mathrm{ob-}$ tained from QSM computations for path 2 (all QM atoms). This small difference is due to the fact that QSM does not have an explicit transition state (TS) optimizer [47]. Nevertheless, the agreement for the structures and the barriers are sufficient for the purpose of comparison. Furthermore, in this case, we are interested in comparing between the different paths calculated with QSM. As shown in Figure 3 , the path including all charges gives TS that is too early along the reaction coordinate. For the excluded charge path, the shape is similar to the all QM atom path; however, the barrier is overestimated. On the other hand, the use of damped charges gives the correct energy, and the correct position for the TS. In all cases the calculated critical points (namely the structures of the reactants, TS, and products) are very similar for all paths, with deviations less than $0.05 \AA$ (see Fig. 4).

The second test corresponds to the first step of the reaction catalyzed by $4 \mathrm{OT}$. $4 \mathrm{OT}$ is a bacterial enzyme that catalyzes the isomerization of 2-oxo-4hexenedioate to 2-oxo-3-hexenedioate [49] and is part of a metabolic pathway that enables the 

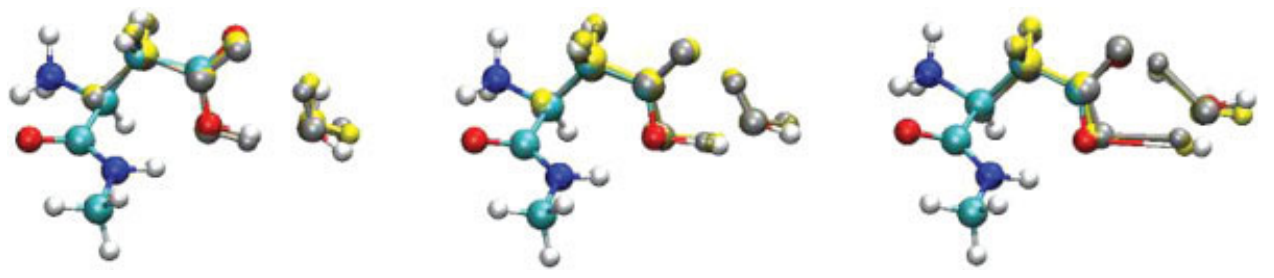

FIGURE 4. Superposition of the calculated critical points along the calculated paths: reactant (left), transition state (middle), and product (right), for the Asp proton transfer reaction. Color code: CPK = all QM atoms; orange = excluded charge; brown = all charges included; silver = damped charges. [Color figure can be viewed in the online issue, which is available at www.interscience.wiley.com.]

Pseudomonas putida bacteria to use aromatic hydrocarbons as its sole source of energy.

We have performed extensive QM/MM studies on this system previously [50-52]. In this case, the setup of the QM and MM subsystems is the same as in our previous studies for the calculations for all three paths. The three paths correspond to: (1) QM/MM QSM path optimization with excluded charges (similar to our previous studies [52]); (2) QM/MM QSM path optimization including all charges in the MM subsystem; and (3) QM/MM QSM path optimization including all charges, with damped charges $\left[\lambda_{\mathrm{AB}}=-5.0\right.$ in Eq. (5)] on the atoms directly bonded to the pseudobond atoms.

Figure 5 shows the three paths calculated for the $4 \mathrm{OT}$ reaction. As expected a large increase in the barrier is observed for path 2, for which all charges are included. This is due to the incorrect polarization of the QM wavefunction by the charges that are in close proximity to the QM/MM boundary. In comparison, the path calculated with damped

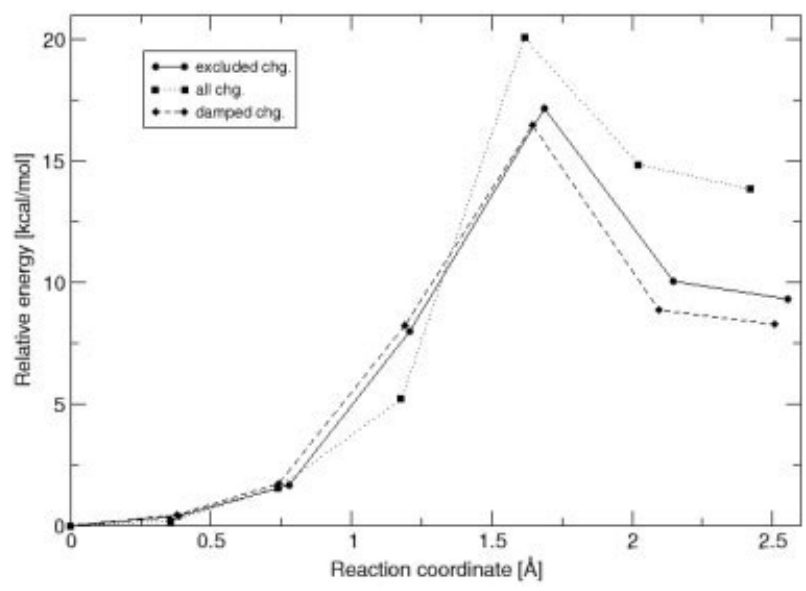

FIGURE 5. Calculated paths for the first step of the 4OT reaction. charges is in good agreement with the conventional $\mathrm{QM} / \mathrm{MM}$ path. This shows that the damped charges indeed provide the correct polarization environment for the QM subsystem, which allows a proper mechanistic description of the reaction.

\section{Conclusion}

Other effects, such as polarization (see Ref. [15a] together with references therein, and Ref. [33]), occurring in intermolecular interactions are implemented in advanced force fields, but here we have shown that a damped charge approach could be of interest for the future development of improved classical force fields. Indeed, we noticed an improvement of the agreement with ab initio calculations, as electrostatics damping allows including part of the penetration energy. The resulting pointcharge energies are better than those obtained using naked "undamped" distributed multipoles. The approach offers increased accuracy and is interesting for the specific case of metal cations. Indeed, limited implementation of damped charges could be easily included in AMBER or CHARMM to improve the cation specificity which is usually completely dependent on the Lennard-Jones parametrization, because, from the sole electrostatic point of view, it is impossible to discriminate between different ions bearing the same charge. Consequently, such simple framework could be the basis for the development of simple and accurate polarizable force fields. Preliminary QM/MM tests have shown that the approach can be in principle employed to include the charges at the boundary, which are usually neglected in QM/MM calculations, by damping the charges close to the boundary without any reparameterization of the van der Waals component of the force field. These results show that the reaction paths computed using damped charges are 
in good agreement with reference QM paths. This last point opens the possibility of a specific parameterization of charges close to QM/MM boundary that could be automatically damped to perform more accurate QM/MM embeddings.

\section{ACKNOWLEDGMENTS}

The advanced biomedical computing center NCI-FCRDC is gratefully acknowledged for computing time. G. A. C. thanks Dr. S. Burger, Dr. Z. $\mathrm{Lu}$, and Prof. W. Yang for the QSM code and helpful discussions. We also thank CRIHAN (Saint-Etienne-du-Rouvray, France) and the CCRE (UPMC, France) for generous allocation of computer time.

\section{References}

1. Case, D. A.; Cheatham, T. E.; Darden, T. A.; Gohlke, H.; Luo, R.; Merz, K. M.; Onufriev, A.; Simmerling, C.; Wang, B.; Woods, R. J. J Comput Chem 2005, 26, 1668.

2. MacKerell, A. D., Jr.; Brooks, B.; Brooks, C. L., III; Nilsson, L.; Won, Y.; Roux, B.; Karplus, M. In The Encyclopedia of Computational Chemistry; Schleyer, P. v. R.; Allinger, N. L.; Clark, T.; Gasteiger, J.; Kollman, P. A.; Schaefer, H. F., III; Schreiner, P. R., Eds.; Wiley: Chichester, 1998; Vol. 1, p 271.

3. van Gunsteren, W. F.; Berendsen, H. J. C. Angew Chem Int Ed Engl 1990, 29, 992.

4. Bayly, C. I.; Cieplak, P.; Cornell, W. D.; Kollman, P. A. J Phys Chem 1993, 97, 10269

5. Singh, U. C.; Kollman, P. A. J Comput Chem 1984, 5, 129.

6. Essmann, U.; Perera, L.; Berkowitz, M. L.; Darden, T. A.; Lee, H.; Pedersen, L. G. J Chem Phys 1995, 103, 8577.

7. Darden, T. A.; York, D.; Pedersen, L. G. J Comput Chem 1993, 98, 10089.

8. Warshel, A.; Levitt, M. J Mol Biol 1976, 103, 227.

9. Lin, H.; Truhlar, D. Theor Chem Acc 2007, 117, 185.

10. Stone, A. J.; Aderton, M. Chem Phys Lett 1982, 83, 233.

11. Vigné-Maeder, F.; Claverie, P. J. Chem Phys 1988, 88, 4934.

12. Cisneros, G. A.; Piquemal, J.-P.; Darden, T. A. J Chem Phys 2006, 125, 184101.

13. Kosov, D.; Popelier, P. L. A. J Chem Phys 2000, 113, 3969.

14. Piquemal, J.-P.; Chevreau, H.; Gresh, N. J Chem Theory Comput 2007, 3, 824.

15. (a) Gresh, N.; Cisneros, G. A.; Darden, T. A; Piquemal, J.-P. J Chem Theory Comput 2007, 3, 1960; (b) Gresh, N.; Piquemal, J.-P. SIBFA, Sum of Interactions Between Fragments Ab initio computed, Version 2007; University Paris 5/Universty Paris 6/CNRS: France, 2007.

16. Liem, S. Y.; Popelier, P. L. A. J Chem Phys 2003, 119, 4560.

17. Ren, R.; Ponder, J. W. J Phys Chem B 2003, 107, 5933.

18. Piquemal, J.-P.; Perera, L.; Cisneros, G. A.; Ren, P.; Pedersen, L. G.; Darden, T. A. J Chem Phys 2006, 125, 054511.

19. Gordon, M. S.; Freitag, M.; Bandyopadhyay, P.; Jensen, J. H.; Kairys, V.; Stevens, W. J. J Phys Chem A 2001, 105, 293.
20. Piquemal, J.-P.; Gresh, N.; Giessner-Prettre, C. J Phys Chem A 2003, 107, 10353.

21. Freitag, M. A.; Gordon, M. S.; Jensen, J. H.; Stevens, W. J. J Chem Phys 2000, 112, 7300.

22. Slipchenko, L. V.; Gordon, M. S. J Comput Chem 2007, 28, 276.

23. Qiang, W. L.; Krimm, S. J Mol Struct (Theochem) 2006, 766, 93.

24. Spackman, M. A. Chem Phys Lett 2006, 418, 158.

25. Rafat, M.; Popelier, P. L. A. J Chem Phys 2005, 123, 204103.

26. Piquemal, J.-P.; Cisneros, G. A.; Reinhardt, P.; Gresh, N.; Darden, T. A. J Chem Phys 2006, 124, 104101.

27. Cisneros, G. A.; Piquemal, J.-P.; Darden, T. A. J Phys Chem B 2006, 110, 13682.

28. Cisneros, G. A.; Elking, D.; Piquemal, J.-P.; Darden, T. A. J Phys Chem A 2007, 111, 12049.

29. Tschumper, G. S.; Leininger, M. L.; Hoffman, B. C.; Valeev, E. F.; Schaefer, H. F.; Quack, M. J Chem Phys 2002, 116, 690.

30. Koenig, P. H.; Hoffman, M.; Frauenheim, Th.; Cui, Q. J Phys Chem B 2005, 109, 9082.

31. Das, D.; Eurenius, K. P.; Billings, E. M.; Sherwood, P.; Chattfield, D. C.; Hodoscek M.; Brooks, B. R. J Chem Phys 2002, $117,10534$.

32. Zhang, Y.; Lin, H.; Truhlar D. G. J Chem Theory Comput 2007, 3, 1378.

33. Giese, T. J.; York, D. M. J Chem Phys 2007, 127, 194101.

34. Bagus, P. S.; Hermann, K.; Bauschlicher, C. W., Jr. J Chem Phys 1984, 80, 4378.

35. Bagus, P. S.; Illas, F. J. Chem Phys 1992, 96, 8962.

36. (a) Piquemal, J.-P.; Marquez, A.; Parisel, O.; Giessner-Prettre, C. J Comput Chem 2005, 26, 1052; (b) Dupuis, M.; Marquez, A.; Davidson, E. R. HONDO 95.3; QCPE, Indiana University: Bloomington, IN, 1995; (c) Gourlaouen, C.; Piquemal, J.-P.; Saue, T.; Parisel, O. J Comput Chem 2006, 27, 142; (d) Gourlaouen, C.; Piquemal, J.-P.; Parisel, O. J Chem Phys 2006, 124, 174311.

37. Lee, C.; Yang, W.; Parr, R. G. Phys Rev B 1988, 37, 785.

38. Becke, A. D. J Chem Phys 1993, 98, 5648.

39. (a) Dunning, T. H., Jr. J Chem Phys 1989, 90, 1007; (b) Rassolov, V.; Pople, J. A.; Ratner, M.; Windus, T. L. J Chem Phys 1998, 109, 1223.

40. Frisch, M. J.; Trucks, G. W.; Schlegel, H. B.; Scuseria, G. E.; Robb, M. A.; Cheeseman, J. R.; Montgomery, J. A., Jr.; Vreven, T.; Kudin, K. N.; Burant, J. C.; Millam, J. M.; Iyengar, S. S.; Tomasi, J.; Barone, V.; Mennucci, B.; Cossi, M.; Scalmani, G.; Rega, N.; Petersson, G. A.; Nakatsuji, H.; Hada, M.; Ehara, M.; Toyota, K.; Fukuda, R.; Hasegawa, J.; Ishida, M.; Nakajima, T.; Honda, Y.; Kitao, O.; Nakai, H.; Klene, M.; Li, X.; Knox, J. E.; Hratchian, H. P.; Cross, J. B.; Bakken, V.; Adamo, C.; Jaramillo, J.; Gomperts, R.; Stratmann, R. E.; Yazyev, O.; Austin, A. J.; Cammi, R.; Pomelli, C.; Ochterski, J. W.; Ayala, P. Y.; Morokuma, K.; Voth, G. A.; Salvador, P.; Dannenberg, J. J.; Zakrzewski, V. G.; Dapprich, S.; Daniels, A. D.; Strain, M. C.; Farkas, O.; Malick, D. K.; Rabuck, A. D.; Raghavachari, K.; Foresman, J. B.; Ortiz, J. V.; Cui, Q.; Baboul, A. G.; Clifford, S.; Cioslowski, J.; Stefanov, B. B.; Liu, G.; Liashenko, A.; Piskorz, P.; Komaromi, I.; Martin, R. L.; Fox, D. J.; Keith, T.; Al-Laham, M. A.; Peng, C. Y.; Nanayakkara, A.; Challacombe, M.; Gill, P. M. W.; Johnson, B.; 
Chen, W.; Wong, M. W.; Gonzalez, C.; Pople, J. A. Gaussian 03, Revision C. 02; Gaussian, Inc.: Wallingford, CT, 2004.

41. Ponder, J. TINKER, Software Tools for Molecular Design, Version 3.6; Washington University: St. Louis, MO, 1998. Updated version available at http://dasher.wustl.edu/ tinker.

42. Cornell, W. D.; Cieplak, P.; Bayly, C. I.; Gould, I. R.; Merz, K. M.; Ferguson, D. M.; Spellmeyer, D. C.; Fox, T.; Caldwell, J. W.; Kollman, P. A. J Am Chem Soc 1995, 117, 5179.

43. Jorgensen, W.; Chandrasekhar, J.; Madura, J.; Impey, R.; Klein, M. J Chem Phys 1983, 79, 926.

44. Zhang, Y.; Lee, T.; Yang, W. J Chem Phys 1999, 110, 46.
45. Zhang, Y. J Chem Phys 2005, 122, 024114.

46. Zhang, Y.; Liu, H.; Yang, W. J Chem Phys 2000, 112, 3483.

47. Burger, S. K.; Yang, W. J Chem Phys 2006, 124, 054109.

48. Gonzalez, C.; Schlegel, H. J Chem Phys 1990, 94, 5523.

49. Whitman, C. P.; Aird, B.; Gillespie, W.; Stolowich, N. J Am Chem Soc 1991, 113, 3154.

50. Cisneros, G. A.; Liu, H.; Zhang, Y.; Yang, W. J Am Chem Soc 2003, 134, 10348.

51. Cisneros, G. A.; Wang, M.; Silinski, P.; Fitzgerald, M. ; Yang, W. Biochemistry 2004, 43, 6885.

52. Cisneros, G. A.; Wang, M.; Silinski, P.; Fitzgerald, M.; Yang, W. J Phys Chem A 2006, 110, 700. 